\title{
A Novel Methodology for Route Stability Using AODV in MANET
}

\author{
Sonia Salaria ${ }^{\mathrm{a}}$, Anil Sagar ${ }^{\mathrm{b}}$, Baljinder Singh $^{\mathrm{c}}$ \\ ${ }^{a}$ Research Scholar, Beant College of Engineering and Technology, Gurdaspur, Punjab, India \\ ${ }^{\mathrm{b}}$ Beant College of Engineering and Technology, Gurdaspur, Punjab, India \\ ${ }^{\mathrm{c}}$ Beant College of Engineering and Technology, Gurdaspur, Punjab, India \\ satbirkaur019@gmail.com
}

\begin{abstract}
A MANET is an autonomous collection of mobile users that communicate over relatively bandwidth restricted wireless links. Since the nodes in topology are mobile, the network might change rapidly and unpredictably with time. The network is decentralized; where all network activities including discovering the topology and delivering messages must be executed by the nodes themselves i.e. route discovering functionality will be incorporated into mobile nodes. But, due to mobility of nodes in a network, the path stability is disturbed and hence the link breakage occurs. In this research work, the signal strength is continuously measured till the node go out of range. Then, time is noticed and if time limit is below threshold value, then the path is not selected. On the other hand, if the value of time limit is above threshold, the path is selected and hence the less breakage occurs in that case.
\end{abstract} $* * * * *$

\section{Introduction}

The decentralized idea of MANET gives extra quality contrasted with the single purpose of disappointment in brought together methodologies, for instance, BS or Access Point. MANETs can be actualized in situation where it is nearly unrealistic to arrange and keep up a wired system (for instance, a shocking zone or a front line). The MANET has specific attributes and confinements:

1. Dynamic topologies: In this system nodes are allowed to move arbitrarily at various paces. So it is hard to foresee the development of a node and its course. In this way, the system topology can change arbitrarily and all of a sudden.

2. Limited data transfer capacity: The wireless associations have less capacity than wired systems. Hotheyver, at present, a few standards offer better information transmission speeds practically identical to Ethernet (for instance, WiMAX). Moreover, ecological conditions, blurring, clamor or impedance influence the execution of wireless correspondence.

3. Battery limitations: In a MANET, nodes can rely upon restricted batteries as a vitality source. Note that a few nodes might not have enough transmit battery to achieve all nodes in the system. This restrains the correspondence of the nodes just to nearby nodes.

4. Media limitations: In a wireless system, it is absurd to expect to transmit and tune in the meantime. Loss of messages is progressively conceivable because of crashes and obstruction.
5. Constrained physical security: in MANET, information packets travel through the air. In this way, it is generally simple for an impostor to find information packets working in free mode and utilizing a bundle tracker. In this manner, sticking or disavowal of administration (DoS) deliberately influences the nodes.

\subsection{Applications of MANETs}

There are numerous MANET applications. The application area for MANET is unique, from little and static systems that are constrained by vitality sources to vast scale and exceedingly powerful versatile systems. Noteworthy precedents incorporate the making of dynamic, productive and survival correspondences for: organize based military/fight conditions, crisis/salvage activities, debacle help tasks, keen transport frameworks, gatherings, portable sensor systems - tolerant, savvy homes, quiet checking, ecological observing and other security-touchy applications. The greater part of these applications require explicit security ensures and solid correspondences. Some realized applications are:

1. Military strategic activities: For the fast and perhaps transient foundation of military interchanges and the organization of troops in unfriendly and/or obscure situations.

2. Pursuit and salvage tasks: For correspondence in territories with insignificant or no remote foundation support.

3. Disaster Relief Operations: For correspondences in conditions where the current foundation is obliterated or left unused.

4. Utilization of the law: For secure and quick interchanges amid law authorization tasks. 
5. Business use: To permit interchanges in displays, gatherings and expansive gatherings. For a few business situations, the requirement for shared preparing might be more vital outside the workplace settings than inside a building. Also, this is regularly the situation when individuals need outside gatherings to coordinate and trade data about a specific undertaking.

\section{Related Work}

[21] Proposed the multiple route routing protocols for ad hoc NETwork mobile (MANET) address the problem of load balancing, scalability, security and network durability. In this document, they propose a new vector distance protocol on demand (SEAM-AODV) ad hoc, multiview of stability and energy efficiency, which is an improvement of the ad hoc distance-on-demand (AODV) protocol. Design a bi-objective optimization formulation to calculate the reliability factor based on stability and residual energy of the nodes, through the cross-layer approach. The reliability of multiple paths is increased through node separation. The route with the highest value of the reliability factor is selected as the main route for data transmission. It also employs an effective path maintenance mechanism to reduce the frequency of route recovery. This protocol is compared to another similar routing protocol: AOMDV. They use ns-2 for the simulation. Our simulation results show that the proposed protocol reduces packet loss up to 25-35\% and routing overhead from about 32 to $40 \%$. It also reaches a package delivery rate of 10 to $13 \%$ higher.

[22] Reported that providing quality service support (QoS) for ad hoc networks is a difficult task due to the dynamism, lack of centralized control, limited resources and the unreliable nature of radio channels and in Mobile Ad hoc. Finding a path that remains valid for a period of networks long enough is also a fundamental task in MANET. This paper proposes a new routing protocol based on QoS and energy stability, which is an extension of our route based on energy and path stability (RSEA), to support applications with performance limitations and delays. In this model, the reliability factor calculated based on the path stability and the residual energy of the intermediate nodes that satisfy admission control. The path with the highest reliability factor is selected to transfer data from the source to the destination among all possible paths. The proposed model shotheyd better results in terms of package delivery ratio, control overhead and end-to-end delay compared to RSQR, LEAR.

[23] Said, in wireless networks, the demand for multimedia data communication is growing. Multicast networks help improve network performance by transmitting data to multiple receivers in a single instance in terms of bandwidth consumption, node battery, and network congestion. Due to the high mobility in ad-hoc mobile networks (MANET), the link error occurs more frequently. In this document, they try to solve the problem or the failure of the multicast network path by selecting stable links in the route building process. Here, they are selecting with the maximum signal strength at the time of data transmission. The high signal strength shows a greater speed of node availability in the communication flow. They have expanded the multicast routing protocol (ODMRP) request for existing media data communication to resolve path and link failure. The basic protocols are processed in the first order of arrival (FCFS). Increase the possibility of road faults. They compared our extended with ODMRP in the Exata / 2.0 simulator of the computer network and to evaluate the packet ratio delivery and the average delay from one end to the other end protocol.

[24] Said, the ad hoc mobile network to (MANET) contains mobile nodes can exchange information bettheyen them without the need for a frame. The development of the nodes is arbitrary; In this way, MANET has a dynamic topology. For this reason, connection breaks in these systems are common. This problem causes a great loss of information and postponement. To reduce this problem, they have reached the possibility of P-AODV. In P-AODV the routes are repaired in the priority premise. In this article the new approach to P-AODV modified routes that are predicted based on the received signal and the energy of the node becomes clear. Nodes that have good RSSI and energy receive high priority and remains of other nodes receive low priority. The accessibility of a neighboring high priority is required for the execution of MP-AODV. This new mechanism reduces delay and packet loss occurs in the original protocol.

[25] Decided to avoid route breaks in the ad hoc mobile network (MANET) by routing data packets using the ad hoc distance carrier routing protocol based on signal strength (RSS). The path hop is predicted using the RSS parameter and the density of the nodes. The intermediate nodes repair the route locally if there are paths an error occurs in the network. The intensity of the received signal indicates the battery level in the received signal. It is used to estimate the intensity of the received signal. If it is low it means that the link should be broken. The density of the nodes is used to describe the number of nodes available within the communication interval. The increase in the number of neighboring nodes increases the transmission delay when they compete to get the channel. Therefore, the proposed scheme reduces transmission delay and avoids route cuts in the network.

[26] Proposed that an important problem for the ad hoc mobile network is the design of the routing protocol. Due to frequent changes in the network topology, this becomes an 
important technical challenge. The failure of the nodes and the breaking of the connections in the network can cause the loss of network resources. In this work they proposed a method that works in two phases; first it is AODV based on signal strength if this approach does not work, so it changes in the second phase means it works like normal AODV. The AODV protocol based on the signal strength measures the signal strength bettheyen the nodes and compares it with the RSSI threshold values if it is greater than the threshold value. it is accepted for further processing; otherwise, it is discarded if no route is found bettheyen the source and destination, then it changes to the normal AODV, which selects the path based on the minimum number of hops. The advantage of this scheme is that by selecting a safe route to the destination, they can increase the useful life of the network. The simulation results show that IAODV performs better than the AODV routing protocol in terms of metrics: packet delivery report, performance, routing overload.

[27] Said, the routing protocol project is an important issue for the ad-hoc mobile network. Due to frequent changes in the network topology, this becomes an important technical challenge. Node failure and network breakdown can cause loss of network resources. The AODV protocol based on the signal strength measures the intensity of the b / w node signal and evaluated with RSSI threshold values if greater than the threshold value, then it is usual for more privileges or additional redundant if it does not find any source $\mathrm{b} / \mathrm{w}$ path and the destination then levered normal AODV, thanks to its faction of normal AODV protocol, they proposed a new technique. In this article they have proposed a method in which the node calculates the RSSI value of the adjacent node if it is smaller than the threshold value and then increases linking the fault factor for 1 and calculating the LFF to the destination node with the help of this method . Factor factor link each route is calculated, and these routes have a minimum binding defect factor that is selected as the first route $\mathrm{b} / \mathrm{w}$ destination senders and selects the path at the base of the minimum step count. The results of the simulation indicate that LFAODV has better compliance with SEAODV routing protocol in terms of performance parameters: packet delivery report, performance, routing overload.

[28] Described that the main problem of ad hoc mobile networks is the design of the routing protocol. The network topology changes frequently and this becomes an important technical challenge. The root cause of the loss of network resources is the failure of the nodes and the connection is interrupted in the network. This paper proposes a method based on the selection of paths based on node-based mobility for the stable administration of links, which works in two phases. First of all, the comparative measurement bettheyen the signal strength and the RSSI value is done. If it is greater than the threshold value, it is accepted for further processing; otherwise it is discarded if no path is found if this approach does not work, then it is changed in the second step means that the work works like a normal AODV. The advantage of this scheme is that, by selecting a safe route to the destination, the useful life of the network increases. The simulation results show that SLM-AODV performs better than the AODV routing protocol in terms of metrics: packet delivery speed, performance, end-to-end average delay.

[29] Described that a critical problem in the Ad Hoc mobile network is how to select a reliable path that can last longer because mobility can cause frequent interruptions in radio links. In this article a new mechanism is proposed to estimate the useful life of the link. Newton's interpolation polynomial is constructed according to different reference points to continuously describe the intensity of the received signal; and therefore, with the mean value and interception methods, the optimal reference points are reasonably selected to estimate the useful life of the link. The results of the simulation have shown that the useful life of the link can be predicted accurately, regardless of the mobility model.

[30] Said that, due to the high mobility of the nodes, the biggest challenge of ad hoc mobile networks (MANET) are the frequent interruptions in connection that lead to numerous failures of routes and route discovery. This article presents a new algorithm based on the signal strength for forwarding the RREQ (SAODV) to better exploit the knowledge of the adjacent coverage and thus reduce the routing overload in the MANET. The results of the simulation confirm the efficiency of our proposed scheme in terms of overload of the RREQ packets, proportion of packet delivery, performance and, finally, the average endto-end delay.

\section{Simulation Scenario and Results}

A detailed simulation model based on NS2 is used. The distributed coordination function (DCF) in 802.11 is used as the MAC layer protocol. 802.11 DCF uses Request To Send (RTS) and Clear To send (CTS) to transmit unicast data to neighboring nodes. The RTS / CTS exchange precedes data transmission and performs a virtual discovery and backup reserve to reduce the hidden terminal problem in wireless networks. CSMA / CA is used to transmit data through the medium. Wave LAN is modeled as a shared medium with a nominal bit rate of $2 \mathrm{Mb} / \mathrm{s}$ with a radio range of 250 meters. 
Table 1 Simulation Parameters

\begin{tabular}{|c|c|}
\hline Parameter & Value \\
\hline Dimension & $1000 \times 1000 \mathrm{~m}^{2}$ \\
\hline Number of Nodes & 50 \\
\hline Simulation Time & $200 \mathrm{~s}$ \\
\hline Traffic Type & $\mathrm{CBR}$ \\
\hline $\begin{array}{c}\text { Number of } \\
\text { Connections }\end{array}$ & 10 \\
\hline Packet Size & 512 bytes \\
\hline $\begin{array}{c}\text { Propagation Radio } \\
\text { Model }\end{array}$ & Two Ray Ground \\
\hline
\end{tabular}

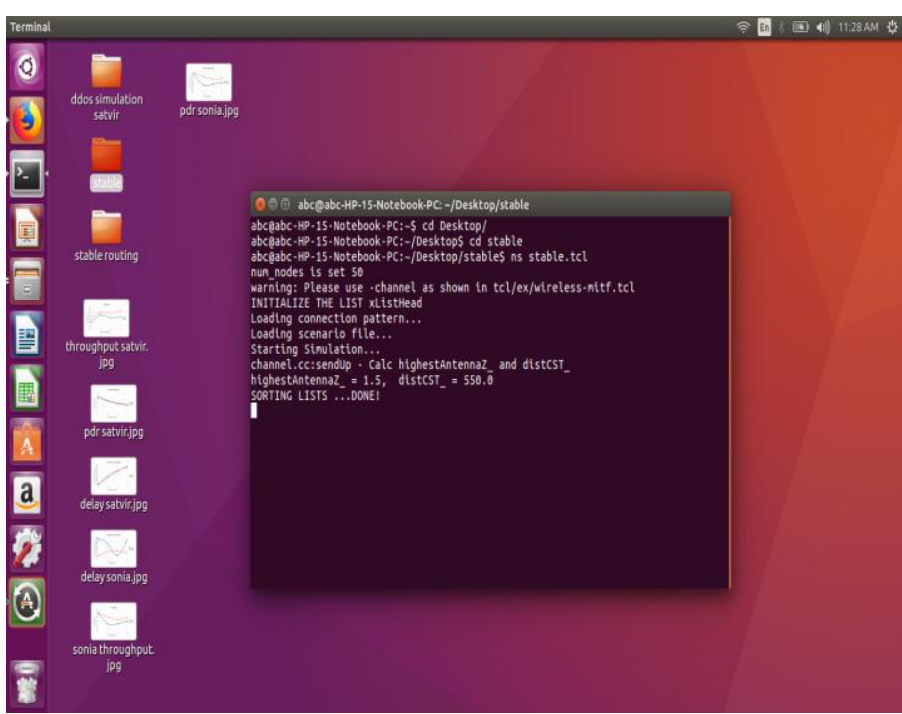

Fig. 1 Simulation Start

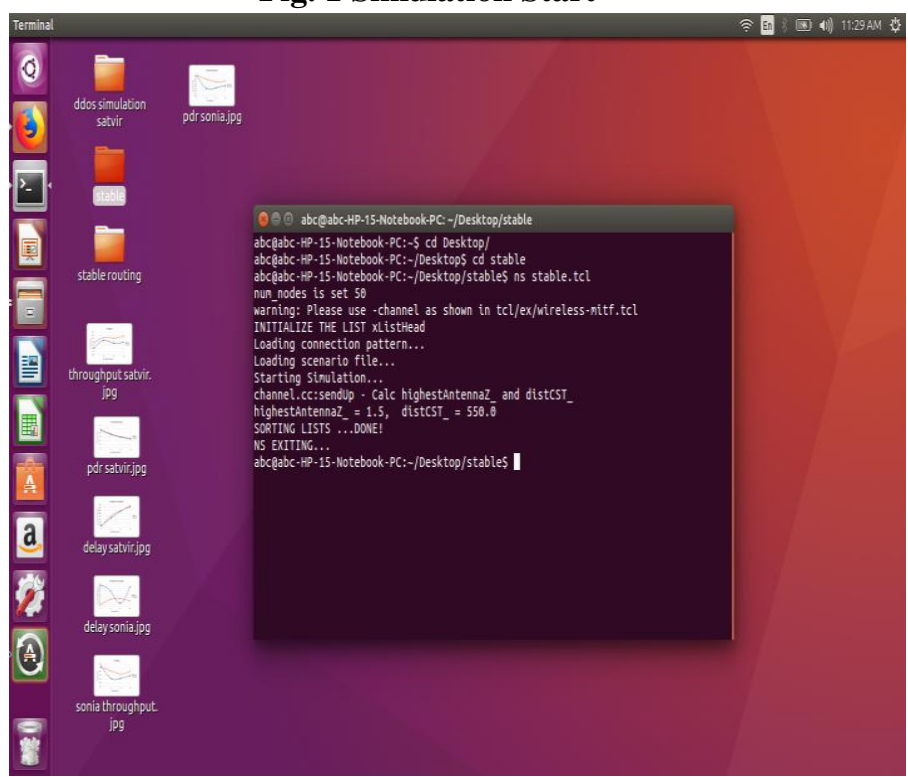

Fig. 2 Simulation Complete

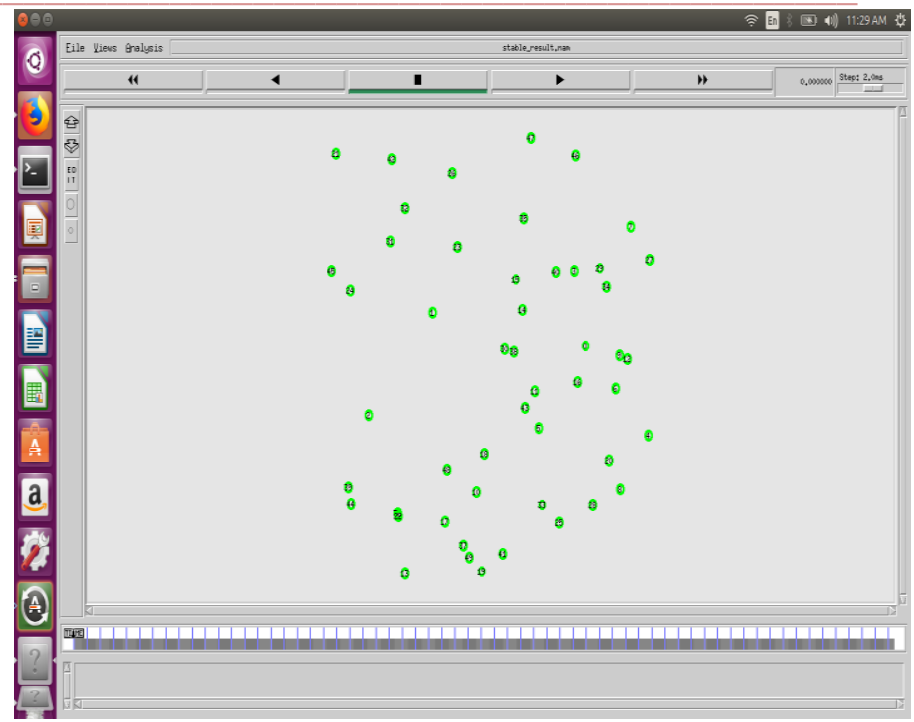

Fig. 3 Number of nodes in a scenario

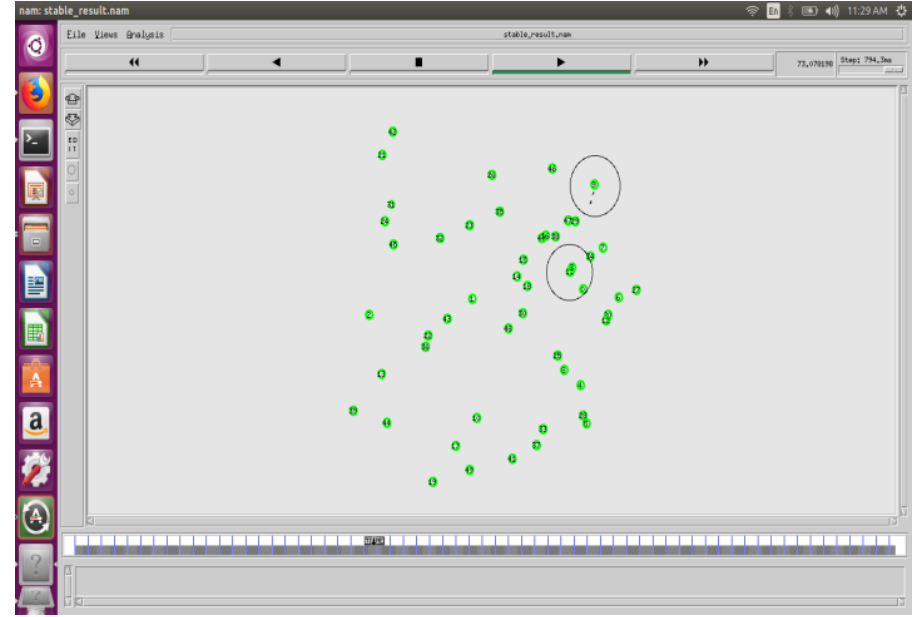

Fig. 4 Running Scenario in NS2

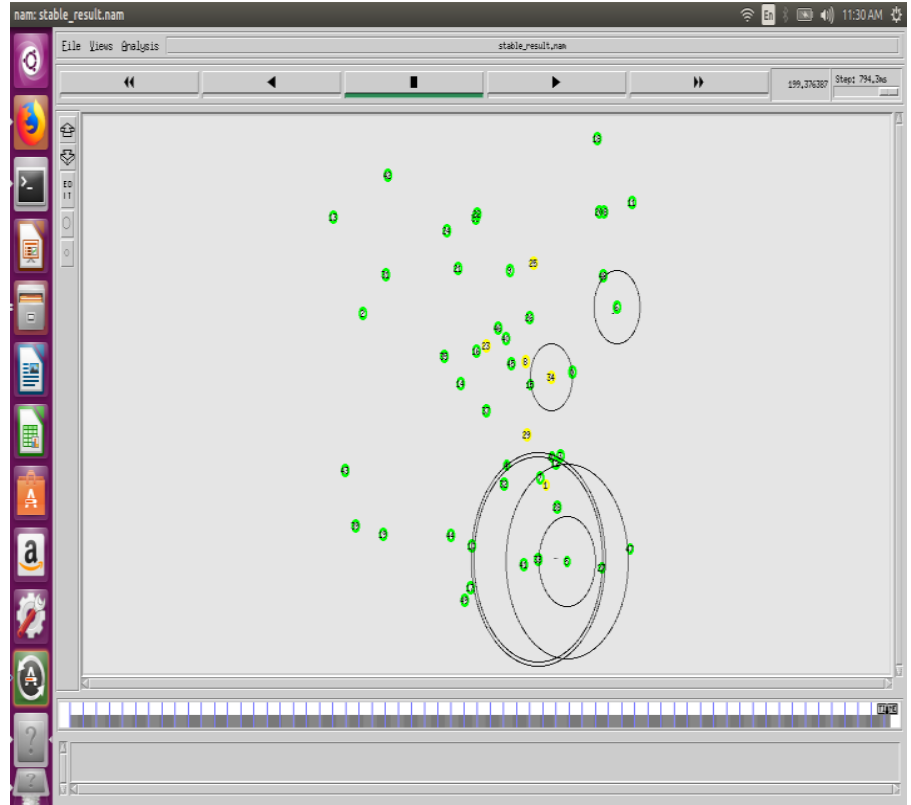

Fig. 5 Nam Console for animation of the Simulation complete 
Traffic sources with bit rates (CBR) are used. Originating destination pairs are randomly distributed across the network. Data packets of 512 bytes are used. The mobility model used is a random reference point in a rectangular area of 1000 X 1000 with 50 knots. The node moves with a mobility of dispositions towards a destination in which it stops for a period of time (pause time) and then moves to the next destination. Simulation execution is 200 simulated seconds. The detailed description of the simulation scenario is shown in Table 1

\section{Performance and Results}

Performance comparison of Base and Stable

1. Packet Delivery ratio: The package distribution relationship in this simulation is defined as the ratio bettheyen the number of packets sent by the constant bit rate sources (CBR, application level) and the number of receiving packets per $\mathrm{CBR}$ receiver in the destination. Specifies the packet loss rate, which limits the maximum network performance.

Table 2. Packet Delivery Ratio(PDR)

\begin{tabular}{|c|c|c|}
\hline Node Mobility & Base Work & Stable \\
\hline 5 & 0.9918 & 0.9922 \\
\hline 10 & 0.9409 & 0.9783 \\
\hline 15 & 0.9435 & 0.9671 \\
\hline 20 & 0.9518 & 0.97 \\
\hline
\end{tabular}

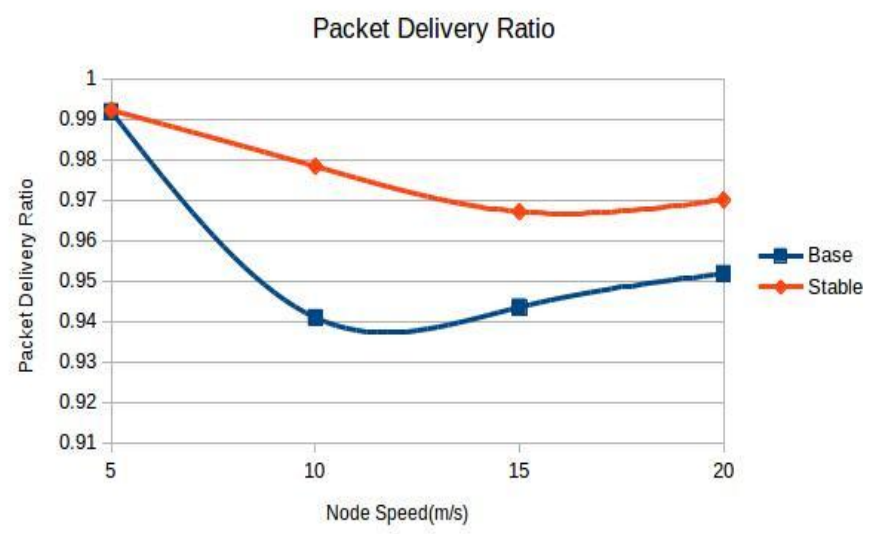

Fig. 6 Packet Delivery Ratio(PDR)

2. End to end Delay: This metric represents an average end-to-end delay and indicates how long it takes for a packet to travel from the source to the target application level. It includes all the possible delays caused by buffering during the latency of the route detection, transmission delays in the
MAC, the queue in the interface queue and the propagation and transfer time. It is measured in seconds.

Table 3: Average End to End Delay

\begin{tabular}{|c|c|c|}
\hline Node Mobility & Base Work & Stable \\
\hline 5 & 189.495 & 22.4939 \\
\hline 10 & 139.877 & 66.5259 \\
\hline 15 & 32.012 & 74.1371 \\
\hline 20 & 176.96 & 51.4188 \\
\hline
\end{tabular}

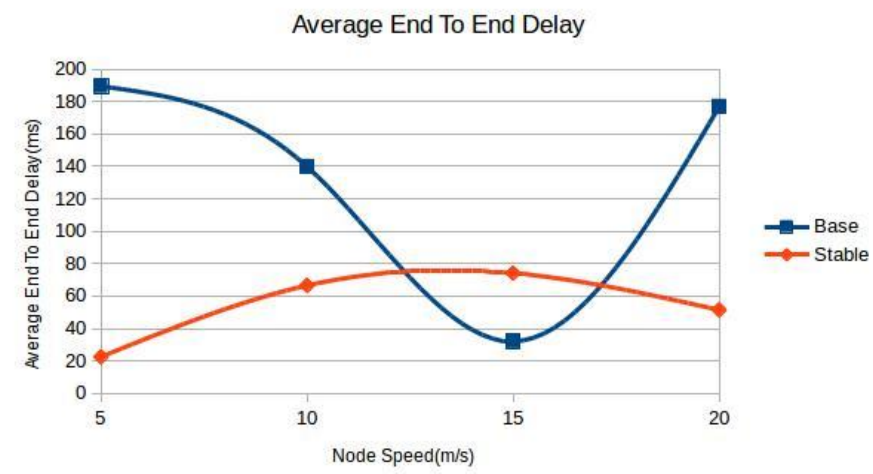

Fig. 7 Average End to End Delay

3. Throughput: Throughput is total packets success fully delivered to individual destinations over total time.

Table 4: Throughput

\begin{tabular}{|c|c|c|}
\hline Node Mobility & Base Work & Stable \\
\hline 5 & 124.76 & 123.99 \\
\hline 10 & 118.18 & 122.28 \\
\hline 15 & 118.25 & 120.64 \\
\hline 20 & 119.68 & 121.97 \\
\hline
\end{tabular}

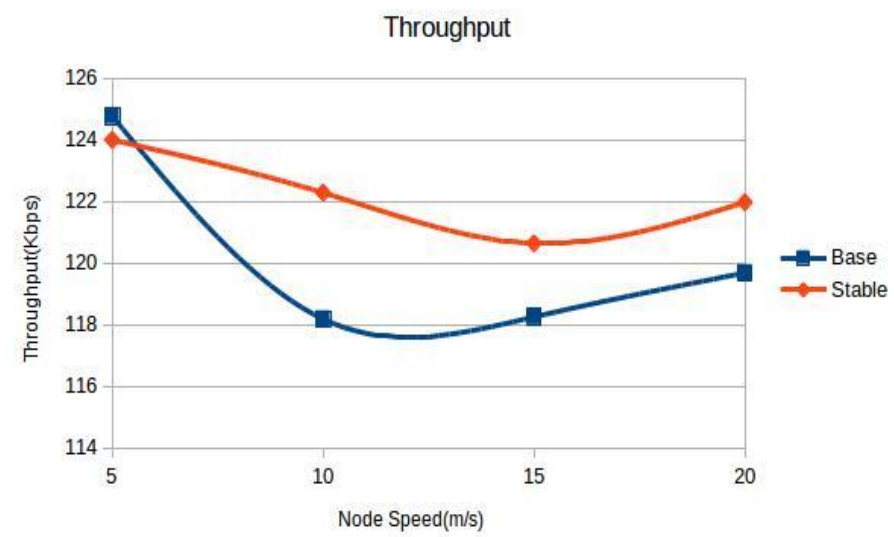

Fig. 8 Throughput 


\section{Conclusion}

MANETs can be placed, where it is reasonably impossible to set up and defend a wired network. In this research work, the signal strength is continuously measured till the node go out of range. Then, time is noticed and if time limit is below threshold value, then the path is not selected. On the other hand, if the value of time limit is above threshold, the path is selected and hence the less breakage occurs in that case. Further, the numerical outcomes indicate that the proposed approach is quite effective to overcome the link breakage conditions in case of MANETs. Hence it also maintains the reliability and efficiency of a network.

[1] P.Srinivasan, P. Kamalakkannan, and S.P.Shantharajah,Ph.D, " Stability and Energy Aware Multipath Routing for Mobile Ad Hoc Networks," International Journal of Computer Applications (0975 8887) Volume 74- No. 2013.

[2] P. Srinivasan and P. Kamalakkannan, "REAQ-AODV: Route Stability and Energy Aware QoS Routing in Mobile Ad hoc Networks," IEEE- Fourth International Conference on Advanced Computing, ICoAC 2012 MIT, Anna University, Chennai. 2012.

[3] S. Brahmbhatt, A. Kulshrestha and G.Singal, "SSLSM: Signal Strength based Link Stability estimation in MANETs," International Conference on Computational Intelligence and Communication Networks, 2015.

[4] S. Mhatre, H. Patil and S. Kadam, "Performance Analysis of Prediction and Priority based Routing Protocol for MANET's," IEEE International Conference on Battery, Control, Signals and Instrumentation Engineering (ICPCSI), 2017.

[5] R. P. Modi and K. Sikamani, "Minimization Of Link Failure In Mobile Ad Hoc Network By Using RSS Based AODV Routing Protocol," International Conference on Current Trends in Engineering and Technology, ICCTET', 2013.

[6] H. Dandotiya, R. Jain and R.Bhatia, "Route Selection in MANETs by Intelligent AODV," 2013 International Conference on Communication Systems and Network Technologies, IEEE, 2013.

[7] C. Dhakad and A.S. Bisen, "Efficient Route Selection By Using Link Failure Factor In MANET," International Conference on Electrical, Electronics, and Optimization Techniques (ICEEOT), 2016.

[8] G. Singh, S. Kaushal, Harpal and S. Bedi, “ Mobility Based Route Selection on the Basis of Nodes for Stable Link Management," International Journal of Advanced Research in Computer Science and Software Engineering, Volume 4, Issue 9, ISSN: 2277 128X September 2014.

[9] W.D.peng, Z.Jian-er and W. Ru-yan, "Received Signal Strength based Link Lifetime Estimating Mechanism in MANET," Special Research Centre for Optical Internet \& Wireless Information Networks, Chongqing University of Posts and Telecommunication, IEEE, 2013.
[10] N. Nissar, N. Naja and A. Jamali, "A Neighbor Signal Strength based Coverage for Reducing Routing Overhead in Mobile Ad Hoc Networks,” IEEE, 2016. 